\title{
Ectopic Pregnancy- Study of Incidence, Risk Factors, Clinical Presentation, and Associated Morbidity in Tertiary Centre
}

\author{
Arjumand Bano ${ }^{1}$, Shalini Narayan ${ }^{2}$ \\ ${ }^{1}$ Senior Resident, Department of Obstetrics and Gynaecology, Chalmeda Anand Rao Institute of Medical Sciences, \\ Karimnagar, Telangana, India. \\ ${ }^{2}$ Junior Resident, Department of Obstetrics and Gynaecology, Chalmeda Anand Rao Institute of Medical Sciences, \\ Karimnagar, Telangana, India.
}

\section{ABSTRACT}

\section{BACKGROUND}

Ectopic pregnancy is one of the commonest acute abdominal emergencies a gynaecologist has to meet in the day-to-day practice. It is also a matter of great concern that a woman might have to face any time during her child bearing period. It not only threatens the life if not treated effectively in a timely manner, but also tells upon her fertility. Many pathological conditions present a percentage of variables but only few have greater disparity of symptoms, signs, opinions, and reports as ectopic, which has made ectopic pregnancy both an interesting and challenging problem, at times difficult to diagnose and manage. The objective of this study is to determine incidence, risk factors, clinical presentation and associated morbidity of ectopic pregnancy.

\section{METHODS}

The cross-sectional study was carried out in the Department of Gynaecology and Obstetrics of Chalmeda Anand Rao Institute of Medical Sciences, Karimnagar. The study period was two years from March 2017 to February 2019. A total of 52 cases of ectopic pregnancies were studied for risk factors, clinical presentation and associated morbidity during the period of study.

\section{RESULTS}

The incidence of ectopic pregnancies is 1:114. The maximum number of ectopic gestations in the present series occurred between the age group 26 to 30 years and more in nulliparous and in the first and second gravidas. Majority of cases belonged to the low socioeconomic status. In the study, no risk factors were identified in $30.7 \%$ of the cases and various other risk factors were studied in remaining cases. The typical triad of amenorrhea, pain abdomen and bleeding were observed in $74.5 \%$ cases. Most of the patients were referred from outside with diagnosis of ruptured ectopic pregnancy, so treatment modality was surgical- laparoscopic unilateral salpingectomy in $71.1 \%$ cases. No morbidity or mortality were seen in the present study.

\section{CONCLUSIONS}

High index of suspicion is required in women in reproductive age group with lower abdomen pain irrespective of presence or absence of amenorrhea, whether or not she has undergone sterilisation to offer a conservative line of management in case of early diagnosis of ectopic pregnancy.

\section{KEY WORDS}

Ectopic Pregnancy, Incidence, Risk Factors, Clinical Presentation, Morbidity
Corresponding Author: Dr. Arjumand Bano, Senior Resident, Department of Obstetrics and Gynaecology, Chalmeda Anand Rao Institute of Medical Sciences, Karimnagar, Telangana, India. E-mail: arju2574@gmail.com

DOI: $10.14260 /$ jemds/2019/547

Financial or Other Competing Interests: None.

How to Cite This Article:

Bano A, Narayan S. Ectopic pregnancystudy of incidence, risk factors, clinical presentation, and associated morbidity in tertiary centre. J. Evolution Med. Dent. Sci. 2019;8(31):2514-2518, DOI: 


\section{BACKGROUND}

Ectopic pregnancy is one of the commonest acute abdominal emergencies a gynaecologist has to meet in the day-to-day practice. It is also a matter of great concern that a woman might have to face any time during her child bearing period. It not only threatens the life if not treated timely and effectively but also tells upon her fertility. The term ectopic is more comprehensive than extra uterine or tubal pregnancy for it embraces all varieties of gestation outside the uterine cavity, includes fallopian tube, ovarian pregnancy, angular pregnancy, pregnancy in a rudimentary uterine horn, abdominal pregnancy, cervical pregnancy, heterotopic pregnancy, caesarean scar pregnancy ${ }^{1}$ Many pathological conditions present a percentage of variables but only few have greater disparity of symptoms, signs, opinions, and reports as ectopic, which has made ectopic pregnancy both an interesting and challenging problem at times difficult to diagnose and manage.

\section{METHODS}

This cross-sectional study was carried out in the department of Gynaecology and Obstetrics of Chalmeda Anand Rao Institute of Medical Sciences, Karimnagar. The study period was two years from March 2017 to February 2019. Sample size taken was 5928 by convenience sampling technique. Ethics approval was taken from institutional ethics committee. All patients with history suggestive of ectopic pregnancy and in whom diagnosis was confirmed by clinical acumen, ultrasound. Statistical analysis with SPSS software was done.

\section{Inclusion Criteria}

The women who were diagnosed as ectopic pregnancy, who were in reproductive age group, of 15-44 years.

\section{Exclusion Criteria}

All intrauterine pregnancies

On admission, history of the patient was taken in which the following points were noted-

1. Name, age, address, occupation and social status.

2. History of amenorrhea whether present or not.

3. History of pain abdomen acute or vague.

4. History of vaginal bleeding if present, duration and nature.

5. Any attacks of syncope or vomiting.

6. Urinary or rectal symptoms, dysuria or retention.

7. History of fever or untoward symptoms like backache or any other discomfort.

8. History of shoulder pain if present

9. Menstrual history- menarche, menstrual period, cycle pattern, duration of flow, dysmenorrhea, last menstrual period.

10. Obstetrical history- number of conceptions, number of full-term deliveries, date of last child birth, nature of deliveries, puerperium, number of abortions, if any, date of last abortion.

11. History of previous ectopic pregnancy, if present, detailed record.
12. History of previous surgery dilatation and curettage, appendicectomy or any other abdominal surgery.

13. History of pelvic inflammatory disease and treatment received fir it or tuberculosis.

14. Family history of tuberculosis.

15. History of infertility, previous tubal surgeries tuboplasty.

16. Method of family planning - IUCD, mini pill.

\section{General Examination}

Following points were noted-

1. Condition of the patient - anaemia, presence of shock, restlessness, cold and clammy extremities.

2. Pulse, respiration, blood pressure and temperature.

3. Cardiovascular and respiratory systems were also examined.

\section{Per Abdomen Examination}

1. Guarding, rigidity, tenderness.

2. Presence of mass, signs of free fluid in peritoneal cavity.

3. Presence of rare signs like Cullen's signs.

\section{Vaginal Examination}

1. Bleeding - nature.

2. Colour of the cervix.

3. Tenderness on movement of the cervix.

4. Position of the cervix whether pulled or not.

5. Size of the uterus, mobility and consistency.

6. Presence of mass in any of the fornices.

\section{Per Rectal Examination}

Was done for confirmation of findings whenever necessary.

\section{Investigations}

- HB\%, blood grouping and rh typing.

- Urine pregnancy test.

- Ultrasonography.

\section{RESULTS}

Total number of deliveries in obstetrics and gynaecology department in Chalmeda Anand Rao Institute of Medical Sciences during this study period were 5928. Total number of ectopic pregnancies during this period of study were 52 . Therefore, the incidence of ectopic pregnancies being 1:114 (Table 1)

\begin{tabular}{|c|c|}
\hline Diagnosis & Number of Cases \\
\hline Ectopic pregnancy & 52 \\
\hline Number of pregnancies & 5928 \\
\hline Incidences & $1: 114$ \\
\hline \multicolumn{2}{|c|}{ Table 1. No. of Cases } \\
\hline
\end{tabular}

\begin{tabular}{|c|c|c|}
\hline Age Group & No. of Cases & Percentage \\
\hline $15-20$ & 6 & 11.5 \\
\hline $21-25$ & 19 & 36.5 \\
\hline $26-30$ & 22 & 42.3 \\
\hline $31-35$ & 4 & 7.6 \\
\hline $36-40$ & 1 & 1.9 \\
\hline Total & $\mathbf{5 2}$ & $\mathbf{1 0 0}$ \\
\hline \multicolumn{2}{|c|}{ Table 2. Age Group Relation to Ectopic Pregnancy } \\
\hline
\end{tabular}




\begin{tabular}{|c|c|c|}
\hline Parity & No. of Cases & Percentage \\
\hline Nulliparous & 20 & 38.4 \\
\hline 1 & 15 & 28.8 \\
\hline 2 & 12 & 23 \\
\hline 3 & 4 & 7.6 \\
\hline 4 & 1 & 1.9 \\
\hline Total & $\mathbf{5 2}$ & $\mathbf{1 0 0}$ \\
\hline \multicolumn{2}{|c|}{ Table 3. Parity Relation to Ectopic Pregnancy } \\
\hline
\end{tabular}

\begin{tabular}{|c|c|c|}
\hline SES Category & No. of Cases & Percentage \\
\hline Low & 43 & 82.6 \\
\hline Medium & 9 & 17.3 \\
\hline High & 0 & 0 \\
\hline Total & $\mathbf{5 2}$ & $\mathbf{1 0 0}$ \\
\hline \multicolumn{2}{|l}{ Table 4. SES Relation to Ectopic Pregnancy } \\
\hline
\end{tabular}

\begin{tabular}{|c|c|c|}
\hline Risk Factors & No. of Cases & Percentage \\
\hline None & 16 & 30.7 \\
\hline Oral contraceptives & 3 & 5.7 \\
\hline Tubectomy & 2 & 3.8 \\
\hline Dilatation and curettage & 10 & 19.2 \\
\hline IUCD & 1 & 1.9 \\
\hline Previous abortions & 5 & 9.6 \\
\hline Previous ectopic pregnancies & 2 & 3.8 \\
\hline Infertility & 5 & 9.6 \\
\hline Appendicectomy & 1 & 1.9 \\
\hline Pelvic inflammatory disease & 7 & 13.4 \\
\hline Total & $\mathbf{5 2}$ & $\mathbf{1 0 0}$ \\
\hline Table 5. Risk Factors for Ectopic Pregnancy \\
\hline
\end{tabular}

\begin{tabular}{|c|c|c|}
\hline Procedure & No. of cases & Percentage \\
\hline Unilateral salpingostomy & 6 & 11.5 \\
\hline Laparoscopic unilateral salpingectomy & 37 & 71.1 \\
\hline Open unilateral salpingectomy & 8 & 15.3 \\
\hline Scar ectopic resection & 1 & 1.9 \\
\hline Total & $\mathbf{5 2}$ & $\mathbf{1 0 0}$ \\
\hline Table 6. Procedure Done for Ectopic Pregnancy \\
\hline
\end{tabular}

Table 2 and 3 above shows that the maximum number of ectopic gestations in the present series occurred between the age group 26 to 30 years; the youngest age was 19 years and the oldest was 40 years. The maximum incidence occurred among the nulliparous and in the first and second gravidas. Shows majority of cases belonged to the low socioeconomic status and only $17.3 \%$ belonged to medium socioeconomic status and none of them belonged to high socioeconomic status. Table 5 shows that there were no risk factors identified in $30.7 \%$ of the cases, $19.2 \%$ of the patients had undergone procedures like dilatation and curettage previously. 5.7\% and $1.9 \%$ used contraceptives in the form OCPs or IUCD respectively.

The typical triad of amenorrhea, pain abdomen and bleeding were observed in $74.5 \%$ cases. Abdominal pain was the most significant symptom in $88.4 \%$ of the cases. Most of the patients were referred from outside with diagnosis of ruptured ectopic pregnancy, so treatment modality was surgical. From Table 6 more cases had Laparoscopic unilateral salpingectomy $(71.1 \%)$ followed by unilateral open salpingectomy (15.3\%), unilateral salpingostomy (11.5\%). Blood transfusion was done in $69.2 \%$ of the cases and patients brought in shock were treated with both blood transfusion and plasma expanders.

\section{DISCUSSION}

This cross-sectional study showed that the incidence of ectopic pregnancies among all deliveries in Chalmeda Anand Rao Institute of Medical sciences was 1:114 which is in accordance with study conducted by Rose Jophy et $\mathrm{al}^{2}$ (1.5:100). Ectopic pregnancy can occur from any time from menarche to menopause, in the present study the maximum incidence was seen in the age group between 21 to 30 years $(78.8 \%)$ which is similar to the study conducted by Samiya $\mathrm{Mufti}^{3}(75 \%)$ and in relation to parity the incidence was maximum among nulliparous (38.4\%), parity $1(28.8 \%)$, parity $2(23 \%)$ which is in accordance to study conducted by Shiv $\operatorname{Kumar}^{4}(25 \%$, $27.5 \%, 25 \%$ respectively) but in study conducted by Rose et $\mathrm{al}^{2}$ nulliparous showed no incidence and maximum incidence in parity 2 . There were no risk factors identified in $30.7 \%$ of the cases, $19.2 \%$ of the patients had undergone procedures like dilatation and curettage, 5.7\% used OCPs, 1.9\% used IUCD, $13.4 \%$ had PID who underwent treatment with antibiotics, 9.6\% had infertility of more than 4 years, 3.8\% had previous ectopic pregnancies. Majority of the patients had an ampullary pregnancy and $44 \%$ had isthmal pregnancy.

\section{Histology}

The anatomic arrangement of the tubal musculature [Hortsman 19521] has described distinct layers of musculatures-

a. Sub peritoneal layer, which runs a longitudinal course along the tube.

b. A middle vasomotor layer whose fibers parallel the vessels encircling the tube.

c. An inner layer arranged in spirals, which originate from different directions and intersect at regular intervals.

\section{Functions of the Fallopian Tube}

The regulatory mechanism concerned in the three-day retention of the ovum within the oviduct and the environment provided by the oviduct for the zygote during the interval are still the worthy subjects for continued investigation.

\section{Fertilization}

Fertilization of the ovum occurs in the ampulla of the uterine tube. One spermatozoon pierces the zona pellucida and enters the ovum for the first 7 days does not increase in the overall size. The morula, which is a clump of cells $-0.13 \mathrm{~mm}$ in diameter by the third or fourth day. The morula still covered by zona pellucid. The trophoblasts have the capacity to burrow into other cells with which they come into contact. It is thus prevented from sticking to the tubal epithelium by zona pellucida which disappears on reaching the uterine lumen.

\section{Aetiology of Tubal Pregnancy5,6}

1. Conditions which Retard Passage of the Fertilized Ovum:

a. Pelvic Inflammatory Disease: It is the commonest cause of ectopic gestation. Endosalpingitis causes desquamation of the epithelium with loss of Cilia. Exosalpingitis gives rise to peritubal adhesions, which restrict the peristaltic movements of the tube. According to ACOG (1998), prior PID, especially that caused by Chlamydia Trachomatis is the most common risk factor. Halbrecht, Vago and Stallworthy observed a high incidence of ectopic pregnancy following treatment of tuberculous salpingitis.

b. Congenital Anomalies: Such as diverticula, accessory ostia and atresia of the tube may distort the lumen.

c. Tumours: Small myomas at the junction of the tube and uterus can obstruct the passage of fertilized egg (Allport 1845) or by mucus polyp (Breslau 1863). 
Other intraluminal lesions are tubal dermoid cyst (Zelinger).

d. Psychologic Causes Including Spasm: Tubal motility is regulated by autonomic nervous system, neuroendocrine imbalance might lead to tubal dyskinesia.

e. Surgical Obstruction: Previous operations such as restoring patency or occasionally deliberate attempt to disrupt continuity (tubal ligation, partial resection or fulguration) can give rise to ectopic pregnancy

f. Intrauterine Contraceptive Device (IUCD): Prevalence of $21 \%$ of ectopic pregnancies in Progestasert users against $2.5 \%$ in other IUCD users. This may be due to altered hormonal environment. An increased incidence of ectopic pregnancy has been reported with the use of IUD with and without progesterone.

g. Hormonal Contraception: An increased incidence of ectopic pregnancy has been reported with the use of progestin only contraceptives and after use of postovulatory high-dose oestrogens to prevent pregnancy the "morning after pill.

h. Assisted Reproductive Techniques: Tubal pregnancy is increased following Gamete Intrafallopian transfer (GIFT) and Invitro fertilization. Chen and associates reported 11 ectopic gestations following 1014 IVF cycles, and 3 of 11 were cornual implantations similarly extratubal as well as heterotypic tubal pregnancies are also increased after these procedures, Berliner and associates described a triplet heterotypic pregnancy following ovulation induction and intrauterine insemination. Abdominal pregnancy has been reported following GIFT and IVF12. Cervical pregnancy may be increased after invitro fertilization. Finally, ovarian pregnancy also may be increased after IVF14.

i. Others: After one previous ectopic pregnancy, the chance of another is $7-15 \%$. Peritubal adhesions subsequent to postabortal or puerperal infection, appendicitis or endometriosis may cause tubal kinking and narrowing of the lumen. These may be associated with the slightly increased risk of ectopic pregnancy following previous induced abortion. Finally, previous caesarean delivery has been linked to a small increased ectopic pregnancy risk. The rate of tubal pregnancy has also been reported to be significantly increased, in women with Luteal Phase Defects, with cigarette smoking, and with vaginal douching.

2. Conditions which Increase Tubal Receptivity: Rubin et al noted endometrium in the lumina of the intrauterine portion of the tube in a few cases of ectopic pregnancy. Factors intrinsic in the conceptus-

a. Transmigration of the Ovum: Delay in the passage of fertilized egg has been proposed as a possible cause of extra uterine gestation.

b. Delayed Ovulation: Iffy suggested that abnormal implantation caused by delayed ovulation suppress the next menstrual period and the reflux flow of the menstruum washes the ovum from the uterus back into tube.

c. Fetal Sex, Sperm and Ovum Abnormalities: Certain studies of fetal sex and tubal pregnancy suggested that, abnormally high percentage were male.

\section{Pathological Anatomy}

Although tubal implantation follows a similar pattern to that recognized in the uterine cavity, the difference in decidual response in the tube modifies the invasiveness of the trophoblast. Moreover, ampulla is also the site for fertilization.

\section{Occurrence of Decidua in the Tube and Later Course}

In the tubal variety, the decidua basalis is either absent or poorly developed and the decidua Vera and the decidua capsularis are essentially not existent.

\section{Uterine Changes}

In a study out of 1125 women with a proven ectopic pregnancy, $75 \%$ had a normal sized uterus. Thus, lack of uterine changes does not exclude an ectopic pregnancy

\section{Behaviour of Uterine Mucosa}

If extra uterine pregnancy continues without interruption, uterine decidua exhibits same features as decidua vera of intrauterine pregnancy with absence of thinning and atrophy.

\section{Arias Stella Reaction}

In 1954, Javier Arias-Stella described typical histological changes in the endometrium, marked cellular atypism and mitotic activity occur with extreme glandular proliferation. Cells are vacuolated and foamy with enlarged and hyper chromatic nuclei and bizarre variation in nuclear shape. In some places there are syncytium-like formation of glands with definite loss of cellular polarity. Ectopic pregnancy ${ }^{7}$ incidence after assisted reproductive technology has decreased over time, but factors such as multiple embryo transfer increase the risk of ectopic pregnancy. In case of pelvic inflammatory disease many cases of chlamydia salpingitis are indolent, cases may go unrecognized causing tubal damage and subsequent tubal pregnancy. Recent change in sex life can cause pelvic inflammation and tubal damage in younger age groups causing more incidence of ectopic pregnancy in young, nulliparous or low parity woman ${ }^{8}$. Further induced abortions increase the incidence of pelvic infections which cause scarring within the fallopian tubes ${ }^{9}$. The scar tissue interferes with the motility or flexibility. The presence of post abortion pelvic inflammatory disease is predictive of a greater likelihood of an ectopic pregnancy in the future. In case of history of previous ectopic pregnancy, according to Jeffcoates there is risk which is 15 times greater than the normal woman. Since tubal disease a nearly always bilateral there is a strong tendency for ectopic pregnancy to occur first on one side and then on the other side. The classical signs ${ }^{1}$ like amenorrhea $(82.10 \%)$, pain abdomen (88.40\%), bleeding (53.8\%), others $(13.4 \%)$ were in accordance to the study by Shiv Kumar ${ }^{4}$. As most of the patients were referred from outside with the diagnosis of ruptured ectopic pregnancy, so treatment modality was mainly surgical. Newer techniques like laparoscopic surgery have bought era of conservative surgical management. In this institute $86.4 \%$ were managed by laparoscopic unilateral salpingectomy which is similar to study done by Shraddha Shetty ${ }^{10}(90.3 \%)$. No post-operative morbidity features like wound infection, DIC, renal failure, septicaemia, pulmonary oedema, was seen in any case. No maternal mortality was seen. 


\section{CONCLUSIONS}

There is an increase in the incidence of ectopic pregnancies but decrease in maternal mortality due the advent of TVS scan and beta hCG diagnosis; but the paradox being that most of the patients are being managed as surgical emergencies as they are brought with the diagnosis of ruptured ectopic pregnancy. High index of suspicion is required in women in reproductive age group with lower abdomen pain irrespective of presence or absence of amenorrhea, whether or not she has undergone sterilisation to offer a conservative line of management.

\section{REFERENCES}

[1] Barnhart KT. Clinical practice, ectopic pregnancy. N Engl J Med 2009;361(4):379-87.

[2] Jophy R, Thomas A, Mhaskar A. Ectopic pregnancy-5 years of experience. J Obstet and Gynecol India 2002;52:55-8.

[3] Mufti S, Rather S, Mufti S, et al. Ectopic pregnancy: an analysis of 114 cases. JK-Practitioner 2012;17(4):20-3.
[4] Shivkumar HC, Umashankar KM, Ramaraju HE. Analysis of 40 cases of ectopic pregnancies in tertiary care hospital in South India. Indian J Basic Appl Med Res 2013;3(1):23541.

[5] Shah N, Khan NH. Ectopic pregnancy, presentation and risk factors, J Coll Physcians Surg Pak 2005;15(9):535-8.

[6] Bhavana, Gupta BK, Pathania BK, et al. Risk factors for ectopic pregnancy: a case control study in tertiary care centre. Journal of Dental and Medical Sciences 2014;13(3):23-7.

[7] Majhi AK, Roy N, Karmakar KS, et al. Ectopic pregnancy: an analysis of 180 cases. J Indian Med Assoc 2007;105(6):308, 310, 312 passim.

[8] Shagufta RSM, Samina M, Reyaz AR, et al. Ectopic pregnancy: an analysis of 114 cases. JK - Practitioner 2012;17(4):20-3.

[9] Gaddagi RA, Chandrashekhar AP. A clinical study of ectopic pregnancy. Journal of Clinical and Diagnostics Research 2012;6(5):867-9.

[10] Shetty S, Shetty A. A clinical study of ectopic pregnancy in tertiary care hospital of Mangalore, India. Innovative Journal of Dental and Medical Sciences 2014;4(1):305-9. 